\title{
Response maxima in modulated turbulence. II. Numerical simulations
}

\author{
Anna von der Heydt, ${ }^{1,2, *}$ Siegfried Grossmann, ${ }^{1}$ and Detlef Lohse ${ }^{2}$ \\ ${ }^{1}$ Fachbereich Physik, Philipps-Universität Marburg, Renthof 6, 35032 Marburg, Germany \\ ${ }^{2}$ Department of Applied Physics and J. M. Burgers Center for Fluid Dynamics, University of Twente, \\ 7500 AE Enschede, The Netherlands
}

(Received 13 February 2003; published 15 December 2003)

\begin{abstract}
Numerical simulations of fully developed turbulence driven by a modulated energy input rate or driving force are performed within two dynamical cascade models, the Gkedzer-Ohkitani-Yamada shell model and a reduced wave vector set approximation of the Navier-Stokes equation. The frequency behavior of the system response is studied and compared with predictions from a variable range mean-field theory, which excludes turbulent fluctuations. In agreement with the mean-field approach, we find a constant response amplitude for low driving frequencies and a $1 / \omega$ decay of the amplitude for high frequencies. In the mean-field theory, the finite cascade time scale had led to an oscillating behavior of the response amplitude as a function of the driving frequency. In the simulations of both models we observe the main maximum. The higher maxima and minima are completely washed out by fluctuations, though the statistical properties of the fluctuations are different in the two models.
\end{abstract}

DOI: 10.1103/PhysRevE.68.066302

PACS number(s): 47.27.Eq, 47.27.Gs, 47.27.Jv

\section{MOTIVATION}

Many realistic turbulent flows are subject to modulated driving forces, as, e.g., the atmosphere of the Earth driven by the periodic heating of the Sun or the pulsed flow through a pipeline. Three dimensional turbulence is characterized by an energy cascade from the outer length scale, where the forcing acts, to the dissipative scale, where most of energy is dissipated, see, e.g., Refs. [1,2]. The down-cascading of energy from large to small scales takes a characteristic time $\tau$. In a statistically stationary flow the energy dissipation rate equals the energy input rate. In a situation with time dependent energy input, on the other hand, this statement will only hold on average, whereas the energy dissipation at a certain time $t$ is expected to depend on the energy input at an earlier time due to the finite time delay of the energy transfer.

In a previous work [3] the effect of an energy input rate modulated in time,

$$
e_{i n}(t)=\epsilon_{0}(1+e \sin \omega t),
$$

with a modulation amplitude $e \ll 1$ and a modulation frequency $\omega$, has been studied within a variable range meanfield theory [4]. The response of the system can be observed in the second order velocity structure function of the flow field at the outer length scale $L, D_{L}(t)=\langle\langle[\boldsymbol{u}(\boldsymbol{x}+\boldsymbol{L}, t)$ $\left.\left.-\boldsymbol{u}(\boldsymbol{x}, t)]^{2}\right\rangle\right\rangle=6 u_{1, r m s}$, which is equivalent to the Reynolds number $\operatorname{Re}(t)=u_{1, r m s}(t) L / \nu$ of the flow and the total energy $E(t)=\left\langle\left\langle\boldsymbol{u}^{2}\right\rangle\right\rangle / 2$ of the system. Here, $u_{1, r m s}$ is the rms of one velocity component and $\nu$ is the viscosity. The response follows the oscillation of the energy input rate with almost constant modulation amplitude at low frequencies $\omega$ of the energy input rate, whereas the response amplitude strongly

\footnotetext{
* Present address: Department of Physics and Astronomy, Institute for Marine and Atmospheric Research Utrecht, Utrecht University, Princetonplein 5, 3584 CC Utrecht, The Netherlands.
}

decreases $(\propto 1 / \omega)$ at higher frequencies. The finite energy transfer time $\tau$ plays a crucial role in this theory. This time $\tau$ is the average time the energy stays within the system while it is transported by the interaction cascade from the large eddies towards the small eddies, where it is finally dissipated. This intrinsic time scale of the system is a multiple $a$ of order 1 of the large eddy turnover time $\tau_{L}$, corresponding to the sum over the eddy turnover times on all scales. $\tau^{-1}$ determines the frequency at which the crossover takes place between the regime of constant response amplitude and decreasing amplitude. In addition, it leads to an oscillating behavior of the system response with driving frequency $\omega$, where the maxima and minima are at frequencies connected to the inverse of the energy transfer time $\tau$. In the limit of large frequencies $\omega$, the extrema of the response can be estimated to be at frequencies $\omega_{r} \simeq n(\pi / \tau), n=1,2,3, \ldots$.

Recent experiments on modulated turbulence in a cylinder between two counter-rotating disks [5] provided evidence for the proposed response maxima. In accordance with the predictions from the mean-field theory [3], for small frequencies a constant response amplitude was measured. For large driving frequencies a $1 / \omega$ decay of the velocity response amplitude was observed, again in agreement with the prediction from our mean-field approach. Note here that both the velocity response as well as the energy response are the same up to a factor of 2, in linear order, cf. Sec. VI of Ref. [3].

In the experiments the amplitude of the driving force rather than that of the energy input rate is modulated. Since the energy input rate is not a controlled quantity anymore, it can serve to measure the response of the system. Of course, also within the mean-field theory we can apply a modulated driving force, see Ref. [3]. The main features, the $1 / \omega$ decay of the energy response amplitude for high frequencies and the constant response amplitude for low frequencies, pertain. The response maxima are only slightly shifted in comparison to the case of a controlled and modulated energy input rate. In the case of a modulated driving force, as in the experiments, the energy input rate as a response of the system, also 
shows maxima in addition to the mentioned mean features. These are at the same frequencies as the maxima of the total energy response amplitude.

In the mean-field approach, the (intermittent) fluctuations of the energy and, in particular, of the cascade time $\tau$ are not present. In experiments and numerical simulations these fluctuations are of course present, and they may lead to broader and less pronounced response maxima and minima. Therefore, in this paper we shall study the frequency dependence of the response to a modulated energy input rate into a system where turbulent fluctuations are included. In particular, we shall address the question whether the response maxima and minima can still be well identified in the presence of fluctuations. Furthermore, we not only consider a modulated energy input rate, but also discuss the slightly different case of a modulated driving force in order to compare with the above mentioned experiments.

An appropriate way to numerically study the problem of modulated turbulence would be a direct numerical simulation of the Navier-Stokes equation for this specific timedependent energy input rate. However, as we need high Reynolds numbers to achieve fully developed, isotropic, and homogeneous turbulence and, in addition, need the response of the system as a function of time for a wide range of driving frequencies, the computational demands would be too high. Therefore, we first study the problem within a dynamical cascade model of turbulence, the Gledzer-Ohkitani-Yamada (GOY) shell model [6-13]. With this model, large Reynolds numbers and enough statistics within a reasonable computing time for each driving frequency can be achieved. The GOY model has been successfully used in a study about decaying and kicked turbulence [14]. In addition, to be even closer to a numerical Navier-Stokes simulation and to distinguish between real effects and artifacts of the turbulence model, we follow another approach. We calculate the response of the system to a modulated energy input rate within a reduced wave vector set approximation (REWA) [15-17], where the Navier-Stokes equation is solved on a reduced, geometrically scaling subset of wave vectors. This method is much closer to the Navier-Stokes dynamics than the GOY model, as it contains (i) much more modes than GOY; (ii) it solves the Navier-Stokes equation for those modes and not only a model equation; and (iii) it is three dimensional. By comparing the results of the two models we can systematically study the effect of fluctuations in different models of turbulence.

Our main results are summarized in Figs. 1, 2, and 3. In Figs. 1 and 2, the amplitude $A$ of the energy response is shown as a function of the driving frequency for both the GOY model (Fig. 1) and the REWA simulation (Fig. 2) with a modulated energy input rate. This is compared with the results of the mean-field model with the corresponding parameters, i.e, the same modulation amplitude $e$ and time scale $\tau$. In Fig. 3 the results from the GOY model solutions are shown for a modulated driving force and compared with the mean-field model. In all cases we observe a constant amplitude for low driving frequencies and a $1 / \omega$-decay for high frequencies. This can, in particular, be observed in the compensated plots [parts (b) of all three figures], where $A$, compensated by its asymptotic amplitude, i.e., $A /\left(\omega \tau_{L}\right)^{-1}$,
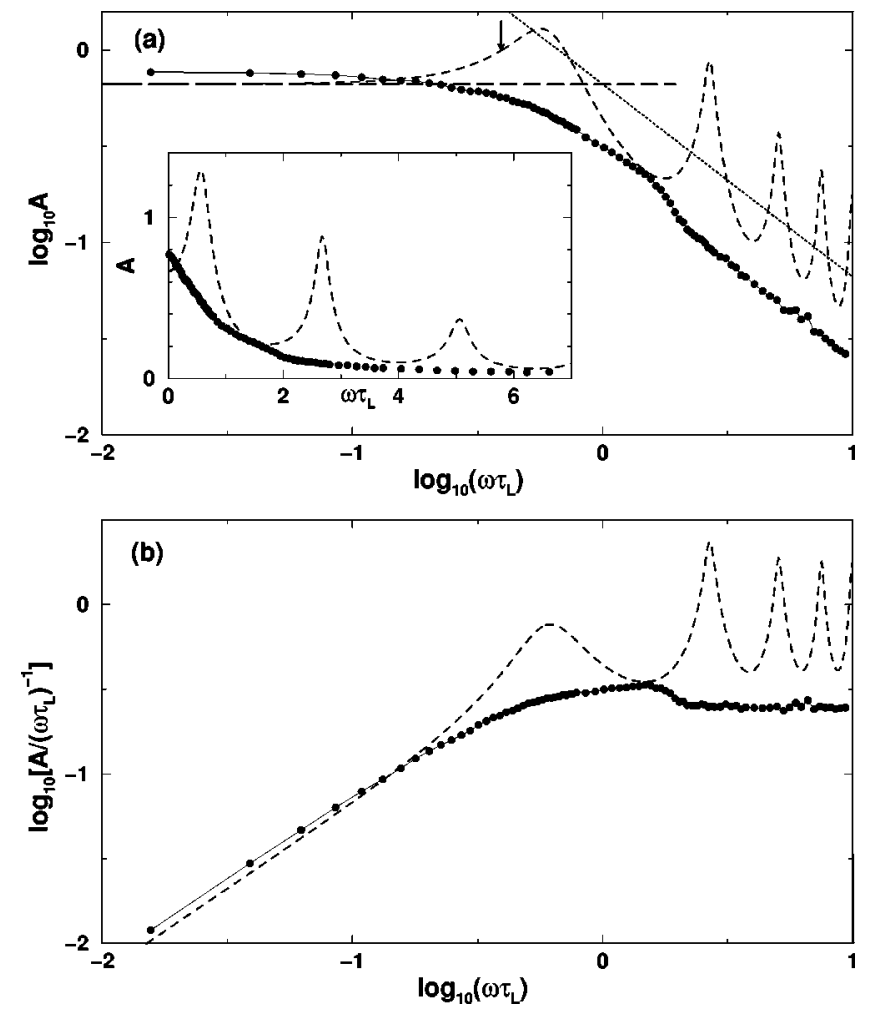

FIG. 1. Response amplitude $A$ as a function of the driving frequency $\omega$ for a modulated energy input rate $e_{i n}=\epsilon_{0}(1+e \sin \omega t)$ calculated within the GOY shell model (full dots), see Sec. II B. The modulation amplitude is set to $e=0.2$, and the cascade time delay turned out to be $\tau / \tau_{L}=a=2.54$. The stationary Reynolds number is $\operatorname{Re}_{0}=7.1 \times 10^{4}$, the viscosity $\nu=1.01875 \times 10^{-4}$, and the large eddy turnover time $\tau_{L}=15.57$. Time and length units are set by $\nu, k_{0}$, and $F_{0}$ in GOY. Our findings are compared with the response amplitude as calculated within the mean-field model with the same $e$ and $\tau$ (dashed lines). (a) Log-log plot of the amplitude $A$ vs frequency. The long-dashed line denotes the low-frequency limit of the mean-field theory, $A \simeq 2 / 3$, and the dotted line the highfrequency limit, $A \propto 2 /(3 \omega)$. The arrow denotes $\omega \tau_{L}=1 / a=0.39$. Near this frequency the crossover takes place in GOY. Inset: linear scale plot of the response amplitude. (b) Log-log plot of the amplitude compensated by the asymptotic amplitude, i.e., $A /\left(\omega \tau_{L}\right)^{-1}$ vs frequency. A clear maximum is observed in GOY at a frequency near the maximum of the mean-field amplitude.

is plotted versus frequency. The $1 / \omega$ decay of the response means that for fast modulation no response is detectable anymore. The remaining dissipation rate is that of the stationary system itself.

In the mean-field approach, a sequence of response maxima is present for both types of forcing, starting at a frequency $\omega \propto 1 / \tau$. In the simulations, this main maximum can also be observed, although it is weaker and broader, i.e., it is "washed out" by fluctuations. The higher-order maxima and minima are not visible in the simulations, but are completely washed out by fluctuations. On the other hand, we emphasize that the turbulent fluctuations in the GOY model are strongly overestimated due to the extreme mode reduction in this model. In the REWA simulation, an artificially large Kolmogorov constant $b$, indicating that still the fluctua- 

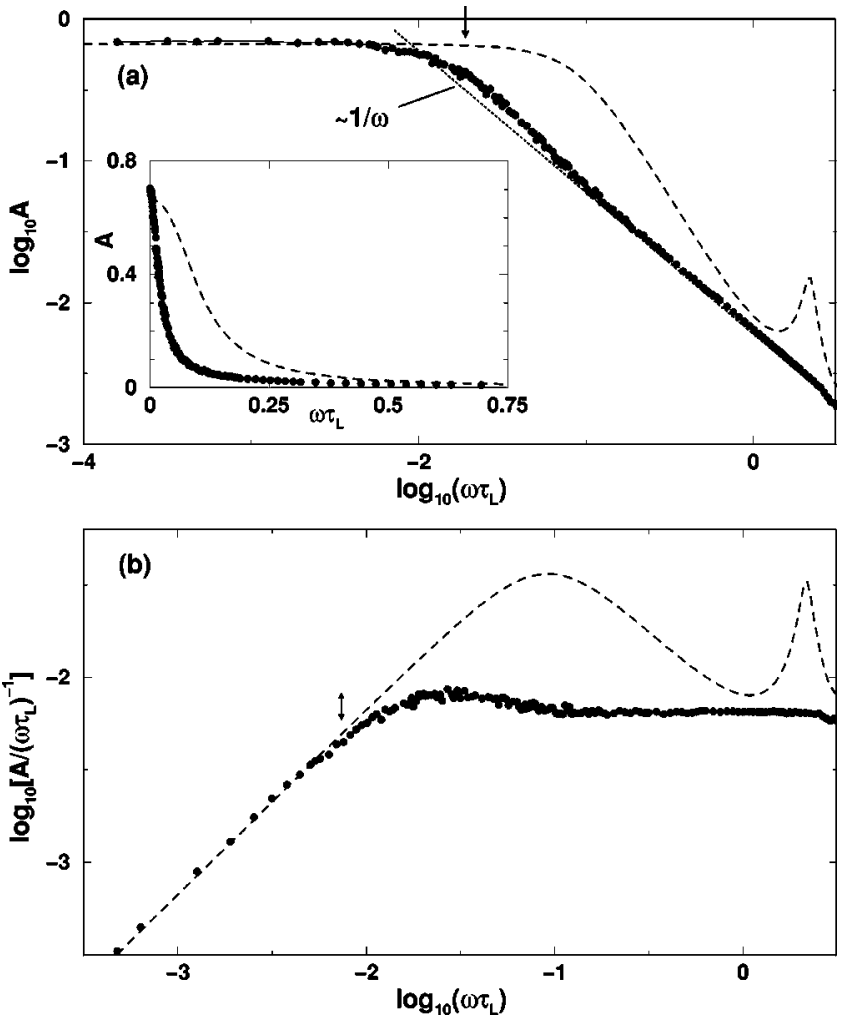

FIG. 2. Response amplitude $A$ as a function of the driving frequency $\omega$ for a modulated energy input rate $e_{i n}=\epsilon_{0}(1+e \sin \omega t)$ calculated within the REWA model (full dots), see Sec. III B. The modulation amplitude is set to $e=0.3$, and the cascade time scale results to be $\tau / \tau_{L}=a=2.94$. The Kolmogorov constant is found to be $b=83.5$ in this simulation instead of $b_{\text {exp }}=6-9$. The stationary Reynolds number is $\operatorname{Re}_{0}=1.2 \times 10^{5}$, the viscosity $\nu=5 \times 10^{-5}$, and the large eddy turnover time $\tau_{L}=0.063$. Times are measured in units of $L_{0}^{2 / 3} \epsilon_{0}^{-1 / 3}$ in REWA. The result is compared with the response amplitude as calculated within the mean-field model with the same $e, \tau$, and $b$ (dashed lines). (a) Log-log plot of the amplitude $A$ vs frequency. The dotted line is $\propto 1 / \omega$. The arrow indicates the mean-field crossover frequency $\omega_{\text {cross }}^{M F} \tau_{L}=(6 / b)^{3 / 2}=0.019$. Inset: linear scale plot of the response amplitude. (b) Log-log plot of the compensated amplitude, i.e., $A /\left(\omega \tau_{L}\right)^{-1}$ vs frequency. A clear maximum is observed in REWA at a frequency near the first maximum of the mean-field amplitude. The arrow indicates the height of the maximum, i.e., a deviation from the $1 / \omega$ decay by a factor of 1.4 in REWA.

tions are stronger than in the Navier-Stokes dynamics, is found. Using such large $b$ in the mean-field approach also leads to a considerable weakening of the first maximum and a shrinking of the higher-order maxima and minima towards very small amplitudes.

These results will be explained and discussed in detail in this paper, which is organized as follows. In the following section we study the modulated turbulence within the GOY shell model. Before calculating the response of the system to a modulated energy input rate as well as a modulated forcing in Secs. II B and II C, we briefly introduce the model and study its stationary properties in Sec. II A. In Sec. III we present our findings on modulated turbulence within the re-
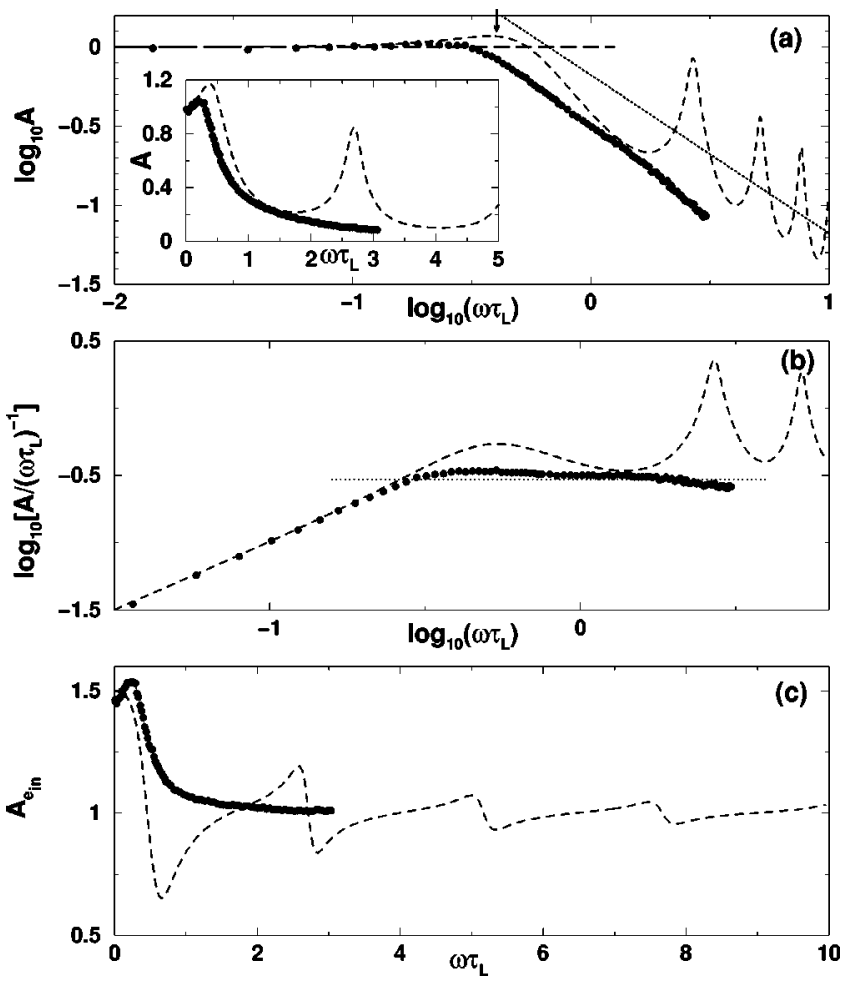

FIG. 3. Response amplitude $A$ and amplitude of the energy input rate $A_{e_{i n}}$ as a function of the driving frequency $\omega$ for a modulated driving force $F=F_{0}(1+e \sin \omega t)$ calculated within the GOY shell model (full dots), see Sec. II C. The modulation amplitude is set to $e_{f}=0.2$, and the cascade time scale is found to be $\tau / \tau_{L}=a=2.48$. The stationary Reynolds number is $\operatorname{Re}_{0}=8.6 \times 10^{4}$, the viscosity $\nu=1.01875 \times 10^{-4}$, and the large eddy turnover time $\tau_{L}=14.5$. The result is compared with the response amplitude as calculated within the mean-field model with the same $e$ and $\tau$ (dashed lines). (a) Log-log plot of the amplitude $A$ vs frequency. The long-dashed line denotes the low-frequency limit of the mean-field theory, $A$ $\simeq 1$, and the dotted line the high-frequency limit, $A \propto 2 /\left(3 \omega \tau_{L}\right)$. The arrow denotes $\omega \tau_{L}=1 / a=0.40$. Near this frequency the crossover takes place in GOY. Inset: linear scale plot of the response amplitude. (b) Log-log plot of the compensated amplitude, i.e., $A /\left(\omega \tau_{L}\right)^{-1}$ vs frequency. The dotted line denotes $A /\left(\omega \tau_{L}\right)^{-1} \propto$ const. (c) Linear scale plot of the energy input amplitude $A_{e_{i n}}$ vs frequency. The mean-field amplitude as well as the GOY amplitude start for low $\omega$ with $A_{e_{i n}} \simeq 1.5$ and merge at $A_{e_{i n}} \simeq 1$ for high frequencies. The GOY amplitude shows only the first main maximum.

duced wave vector set approximation. We summarize our results in Sec. IV.

\section{MODULATED TURBULENCE IN THE GOY SHELL MODEL}

\section{A. Stationary properties}

The GOY shell model consists of a set of coupled ordinary differential equations (ODEs) for one-dimensional complex velocity modes $u_{n}[6-13]$. These modes $u_{n}$ correspond to velocity differences $\left|\boldsymbol{u}\left(\boldsymbol{x}+\boldsymbol{r}_{n}\right)-\boldsymbol{u}(\boldsymbol{x})\right|$ on scale $r_{n} \cdot N$ modes are taken into account, $n=1,2, \ldots, N$, one complex velocity mode per cascade level $n$, defined by the wave num- 
bers $k_{n}=\lambda^{n} k_{0}$ which are equally spaced on a logarithmic scale, here, $\lambda=2$. The model equations read

$$
\begin{aligned}
\left(\frac{d}{d t}+\nu k_{n}^{2}\right) u_{n}= & i\left(a k_{n} u_{n+1}^{*} u_{n+2}^{*}+b k_{n-1} u_{n-1}^{*} u_{n+1}^{*}\right. \\
& \left.+c k_{n-2} u_{n-1}^{*} u_{n-2}^{*}\right)+F \delta_{n, 1}
\end{aligned}
$$

where $n=1, \ldots, N, a=1, b=-1 / 4$, and $c=-1 / 2$. These are the traditional parameters. We impose boundary conditions on the $u_{n}$, i.e., $u_{n}=0$ for $n<1$ or $n>N$. We use $N$ $=14$ shells, a viscosity of $\nu=1.01875 \times 10^{-4}$, and $k_{0}$ $=2^{-4}$. The forcing acts on the largest scale, i.e., the first shell, $n=1 . F$ is constant, $F=F_{0}=(1+i) \times 10^{-2}$. Together with $\nu$ and $k_{0}$, this sets the time and length units as well as the Reynolds number. Equations (2) are integrated using a fourth order Runge-Kutta scheme with adaptive step size [18].

With the above chosen parameters the GOY dynamics is chaotic [13]. The system is forced on large scales while most of the energy is dissipated on small scales. It reaches a steady state, in which the velocities are stochastically fluctuating. In this sense the system has similar properties as three dimensional Navier-Stokes turbulence. The scaling behavior of structure functions and dissipation has been extensively studied in Refs. [10-13,19-21]. The deviations from K41 scaling due to intermittency observed in the GOY model are very similar to experimental values. In order to reduce the computational effort we use only 14 shells, which turns out to be sufficient to achieve an inertial scaling range of about three decades.

The Reynolds number of the system can be defined as follows. An outer length scale $L$ is given by the smallest wave number $k_{1}, L=1 / k_{1}$. A typical velocity $U$ is the velocity on that scale, $\left\langle\left\langle\left|u_{1}\right|^{2}\right\rangle\right\rangle_{t}^{1 / 2}$. The average $\langle\langle\cdots\rangle\rangle_{t}$ is taken over time. With these length and velocity scales the Reynolds number of the present simulation is $\operatorname{Re}_{0}=U L / \nu$ $=8.6 \times 10^{4}$. The simulated time interval is several hundreds of large eddy turnover times $\left|k_{1} u_{1}\right|^{-1}$. The time scales in the model have been determined as follows. For each shell $n$ an eddy turnover time $\tau_{n}$ is defined by [10] $\tau_{n}=1 /\left|u_{n} k_{n}\right|$. This is also considered as the time scale for the turbulent energy transfer through the $n$th level. The time scale relevant for the energy loss on level $n$ due to viscosity is defined as $\tau_{n}^{d}$ $=1 /\left(\nu k_{n}^{2}\right)$. Both time scales are shown in Fig. 4 .

In the inertial subrange (ISR) between shells 2 and 9, where the energy transfer times are the relevant time scales for the dynamics, the decrease of the $\tau_{n}$ with $n$ is near $\tau_{n}$ $\propto 2^{-2 n / 3}$ (dashed line in Fig. 4) as expected for the turnover times of eddies of sizes $r_{n} / L \propto(1 / 2)^{n}$. In this range the dissipation time scales $\tau_{n}^{d}$ are much larger than the $\tau_{n}$, meaning that the turbulent energy transfer is much faster than the viscous dissipation, and therefore the dominant process. In the VSR instead $\tau_{n}^{d}<\tau_{n}$, i.e., on average the energy is dissipated by viscosity before it can be transferred to smaller scales. The largest eddy turnover time is, in general, defined by the velocity on the outer length scale $L$, i.e., on the length scale of the forcing, which in this case is $1 / k_{1}$. However, in this model, the time $\tau_{1}$ is disturbed due to finite size effects.

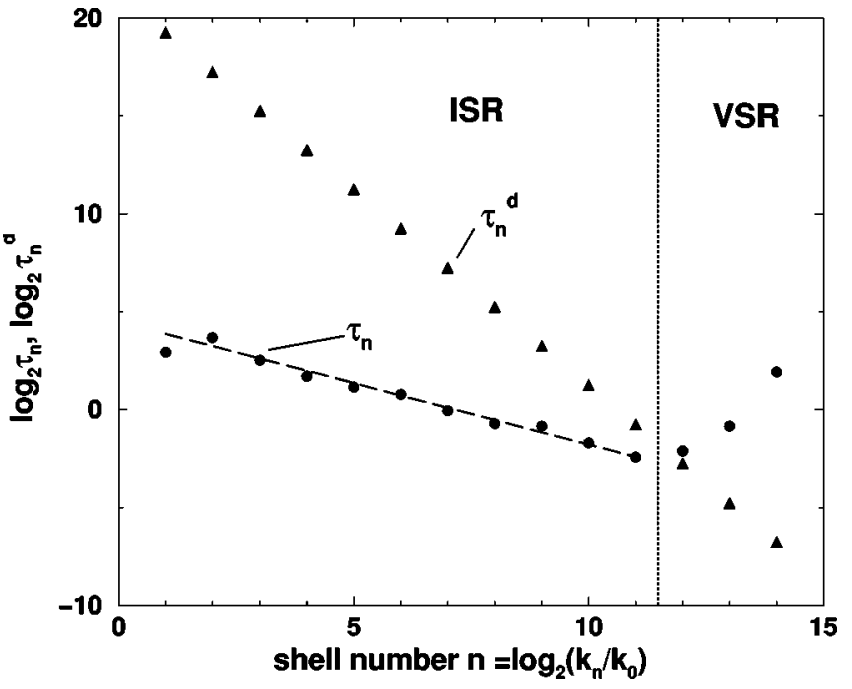

FIG. 4. Characteristic time scales of the turbulent energy transfer $\tau_{n}$ (full dots) and of the viscous dissipation $\tau_{n}^{d}$ (full triangles) as functions of the level number $n$. The dashed line is a linear fit of $\log _{2} \tau_{n}, n=2, \ldots, 11$, and gives $\tau_{n}^{f i t}=22.46 \times 2^{-0.63 n}$. The shown $\tau_{n}$ are obtained by averaging over about 1400 large eddy turnover times. The dotted line indicates the middle of the crossover region between the ISR and the VSR.

Therefore, we extrapolate from the turnover times of the other shells. A linear fit in Fig. 4 for $\log _{2} \tau_{n}$ with $n$ $=2, \ldots, 11$ leads to $\tau_{n}^{f i t}=22.46 \times 2^{-0.63 n}$. Without intermittency one would have $\tau_{n} \propto 2^{-2 n / 3}$; the small deviation corresponds to the intermittent scaling of $u_{n} \propto k_{n}^{-0.37}$ or an intermittency correction of $\delta \xi_{1}=\xi_{1}-1 / 3 \simeq 0.04$. The extrapolation for $n=1$ yields for the large eddy turnover time $\tau_{L}=\tau_{1}^{f i t}=14.5$. The time scale corresponding to the energy transfer time $\tau$ used in the mean-field model [3] is the sum over the eddy turnover times of all energy-input and inertial-range shells, i.e., here $\tau \simeq \sum_{n=1}^{11} \tau_{n} \simeq 35.9$. The factor $\tau / \tau_{L}=a$ between the transfer time and the large eddy turnover time is then $a=2.48$.

Until now, we have considered a constant forcing $F$ $=F_{0}$. The resulting energy input rate $e_{i n}(t)=\left\langle\left\langle u_{1}^{*}(t) F_{0}\right\rangle\right\rangle$ then fluctuates around its mean value because of the $u_{1}^{*}(t)$ fluctuations. In the mean-field theory [4] the energy input rate $e_{i n}$ is constant instead. For closer comparison we also consider another type of forcing in the GOY model: $F(t)$ $=\epsilon_{0} u_{1}(t) /\left|u_{1}(t)\right|^{2}$. This forcing $F(t)$ fluctuates as $u_{1}(t)$. Then the energy input rate is $e_{i n}=\left\langle\left\langle u_{1}^{*} F\right\rangle\right\rangle=\epsilon_{0}=$ const by definition. The ISR-scaling behavior as well as the energy spectrum then turn out to be similar to the previously discussed ones with the constant forcing $F=F_{0}$. The energy transfer time $\tau$ is slightly larger in this case, namely, $\tau$ $=39.5$, and the large eddy turnover time is $\tau_{L}=\tau_{1}^{\text {fit }}$ $=15.57$. Again, the large eddy turnover time is extrapolated from $\tau_{2}, \ldots, \tau_{11}$. This leads to the factor $\tau / \tau_{L}=a=2.54$ between the total time delay of the energy cascade and the large eddy turnover time. In the following sections we will study the time-dependent cases where either the energy input rate $e_{i n}$, i.e., $F=\epsilon_{0} u_{1} /\left|u_{1}\right|^{2}$ (Sec. II B) or the forcing $F$ $=F_{0}$ is modulated (Sec. II C). 


\section{B. Modulated energy input rate}

In this section we apply a modulated energy input rate to the GOY model, i.e., we set the forcing $F=F(t)$ in Eqs. (2) as

$$
F(t)=\epsilon_{0} \frac{u_{1}}{\left|u_{1}\right|^{2}}(1+e \sin \omega t)
$$

with a modulation amplitude $e=0.2$. Then, the resulting energy input rate $e_{i n}$ is

$$
e_{i n}(t)=\left\langle\left\langle u_{1}^{*}(t) F(t)\right\rangle\right\rangle=\epsilon_{0}(1+e \sin \omega t),
$$

and has a prescribed modulation amplitude $e \epsilon_{0}$ by definition. The total energy of the system

$$
E(t)=\frac{1}{2} \sum_{n=1}^{14}\left\langle\left\langle u_{n}^{*}(t) u_{n}(t)\right\rangle\right\rangle,
$$

is calculated for a wide range of driving frequencies $\omega$ in order to study the frequency behavior of the response. The brackets $\langle\langle\cdots\rangle\rangle$ denote the ensemble average. This ensemble average is performed as follows. From a long stationary simulation we collect an ensemble of 1500 starting configurations which we then let evolve according to Eqs. (2) but now including the modulation of the forcing $F(t)$, Eq. (3), and average over these 1500 time series. To ensure that the different realizations can be considered as statistically independent, the time delay between the successive starting configurations for the different realizations is chosen to be about 100 large eddy turnover times.

The oscillating response of the system $\Delta(t)$ is then studied in terms of the ratio between the energy $E(t)$ with modulated energy input and the energy $E_{0}(t)$ without modulation, namely,

$$
E^{n o r m}(t)=\frac{E(t)}{E_{0}(t)}=1+\Delta(t)
$$

$E$ and $E_{0}$ are both averaged over 1500 realizations. In spite of the averaging not only $E$ but also $E_{0}$ still contains (weak) fluctuations. Therefore, we write $E_{0}(t)$, as $E_{0}$ is still slightly fluctuating around its mean value. $E$ and $E_{0}$ contain about the same size of fluctuations. Accordingly, the energy input rate $e_{i n}$ is normalized by its stationary value, $e_{i n}^{\text {norm }}(t)$ $=e_{i n}(t) / \epsilon_{0}=1+e \sin \omega t$.

In Fig. 5 the input rate $e_{i n}^{n o r m}(t)$ and the energy $E^{n o r m}(t)$ are plotted for four different driving frequencies. For the two low frequencies where $\omega \tau_{L} \ll \tau_{L} / \tau=1 / a \simeq 0.39$, the energy follows the oscillation of the energy input rate with almost constant, but smaller, amplitude. For higher frequencies the amplitude of the deviations of the normalized energy from its stationary value 1 strongly decrease, and a phase shift with respect to the energy input becomes visible. The same behavior of the energy has been observed in the mean-field theory [3].

To quantitatively access the frequency behavior of the response amplitude, we calculated time series of the total en-
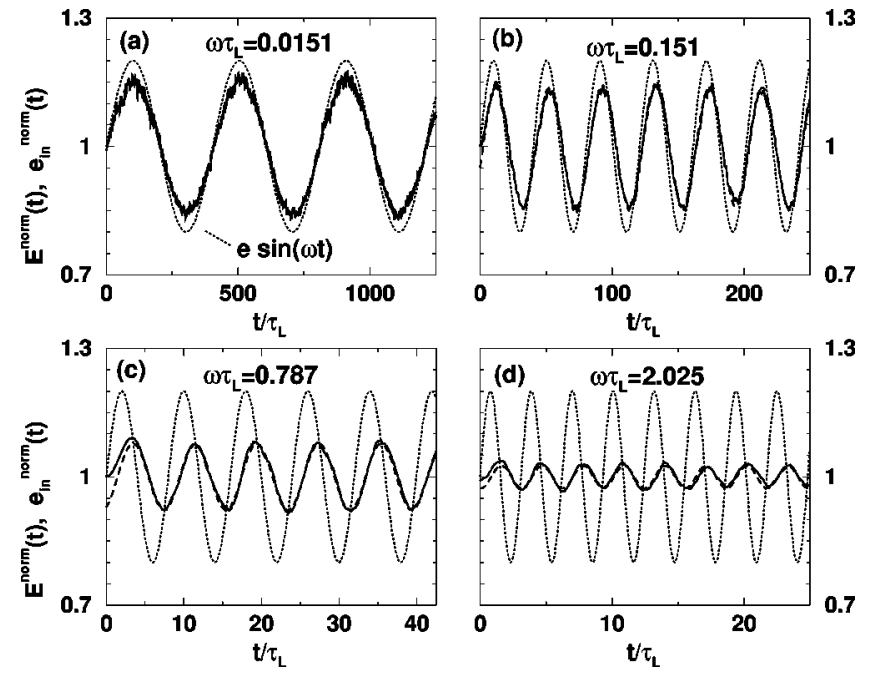

FIG. 5. Energy input rate $e_{i n}^{\text {norm }}=e_{i n} / \epsilon_{0}$ (dotted lines) and energy content $E^{\text {norm }}$ (solid lines) for four different modulation frequencies $\omega$ calculated in the GOY model. The energy input rate $e_{i n}$ is modulated with a modulation amplitude of $20 \%$ of the constant energy input rate $\epsilon_{0}, e=0.2$, according to Eq. (4). Also included is the fit according to Eq. (7) for the energy $E^{\text {norm }}$ (dashed lines, indistinguishable from the solid lines). (a) $\omega \tau_{L}=0.0151$, (b) $\omega \tau_{L}$ $=0.151$, (c) $\omega \tau_{L}=0.787$, (d) $\omega \tau_{L}=2.025$.

ergy $E(t)$ for 85 different driving frequencies varying over almost three decades between $0.012 \leqslant \omega \tau_{L} \leqslant 9.3$. The chosen frequencies are approximately equally spaced on a logarithmic scale. The normalized energy $E^{n o r m}(t)$ is fitted by a function of the form

$$
E^{n o r m}(t)=E_{\text {const }}+e A \sin (\omega t+\Phi)
$$

with three free parameters: $E_{\text {const }}$, the amplitude $A$, and the phase shift $\Phi$. $E_{\text {const }}$ is near 1 for all frequencies, i.e., $E_{\text {const }}=1.0022 \pm 0.0032$. Fits (7) are included in Fig. 5 as dashed lines but they are mostly indistinguishable from the solid lines for the energy itself.

Figure 1 shows the amplitude $A$, resulting from the fitting procedure, as a function of the dimensionless frequency $\omega \tau_{L}$. A is almost constant for low frequencies and has a value of about $2 / 3$. For higher frequencies the amplitude decreases as $\propto 1 / \omega$. The same features have been observed in the mean-field calculations, see dashed lines. The longdashed line in Fig. 1 represents the low-frequency limit of the mean-field theory, $A \simeq 2 / 3$, and the dotted line the highfrequency limit, $A \propto 2 /\left(3 \omega \tau_{L}\right)$.

The crossover between the regime of constant amplitude and the one of $1 / \omega$ decay of the energy response takes place at $\omega_{\text {cross }} \tau_{L} \simeq 1 / a \simeq 0.39$, which is indicated by the arrow in Fig. 1. In the mean-field approach this crossover is always at $\omega \tau_{L}=1$, independent of the factor $a$ between the large eddy turnover time and the total time scale of the energy transfer. In experiments [5] the crossover frequency has been used to measure the energy cascade time scale. The present simulations confirm that this frequency gives the correct order of magnitude for the energy transfer time. 


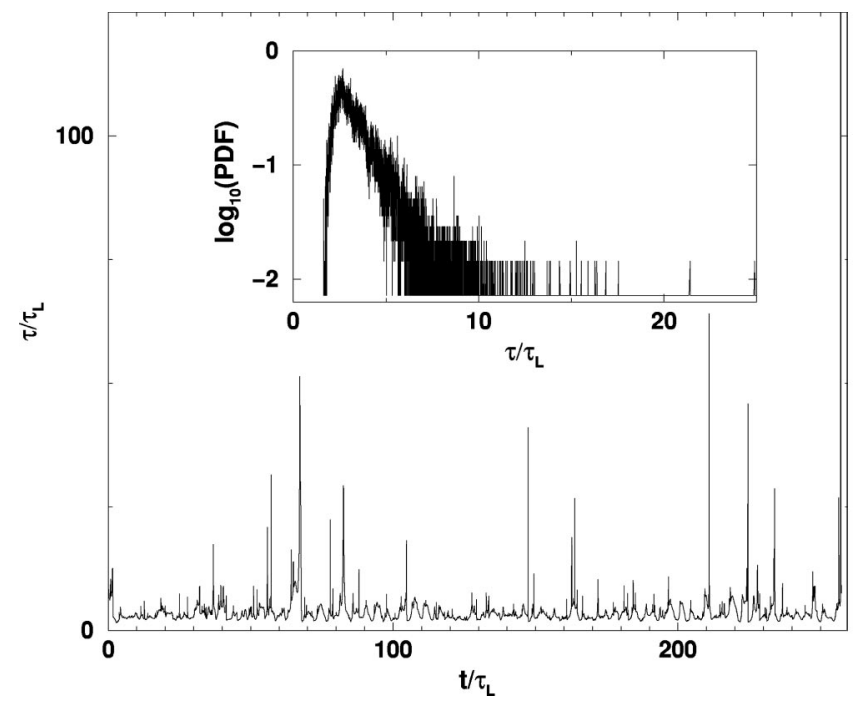

FIG. 6. Time series of the cascade time $\tau(t)$ for nonmodulated forcing $F=\epsilon_{0} u_{1} /\left|u_{1}\right|^{2}$ in the GOY model. Strong fluctuations are observed. Inset: probability distribution of $\tau / \tau_{L}$. The mean is $a$ $=2.54$, and the width $(\mathrm{FWHM}) / 2=0.46 a$ giving about $50 \%$ fluctuations.

Response maxima, as observed in the mean-field model at frequencies connected with the inverse energy transfer time, are difficult to identify in Fig. 1(a). There is some structure visible at $\omega \tau_{L} \simeq 0.31$ and $\omega \tau_{L} \simeq 1.57$. In Fig. 1(b), where the amplitude $A$, compensated by the asymptotic amplitude $\left(\omega \tau_{L}\right)^{-1}$, i.e., $A /\left(\omega \tau_{L}\right)^{-1}$ is plotted vs frequency, this structure becomes more evident, and we see a clear maximum at a frequency of about $\omega \tau_{L} \simeq 1.57$. This maximum probably corresponds to the mean-field maximum. Of course, the maximum in GOY is broadened and weakened due to the large fluctuations, and the higher-order maxima and minima are apparently washed out completely. As in the mean-field theory, no fluctuations are included and the energy cascade time $\tau$ is considered to be constant. However, in the GOY model this assumption is not true, as can clearly be seen in Fig. 6. Here, a time series of the cascade time $\tau(t)$ $=\sum_{n=1}^{11} \tau_{n}(t)$ is plotted, computed within the GOY-shell model with nonmodulated forcing $F=\epsilon_{0} u_{1} /\left|u_{1}\right|^{2}$. The inset shows the probability distribution of $\tau / \tau_{L}$. This distribution has its maximum at $\tau / \tau_{L}=2.39$, almost at the mean cascade time $a=2.54$, and a width of about $0.46 a[(\mathrm{FWHM}) / 2)]$ (FWHM, full width at half maximum). The width is almost half the size of the mean which indicates that the transfer time fluctuates strongly and therefore we have to expect that the response maxima are more or less washed out. However, these strong fluctuations are considered as an artifact of the GOY model and not as a feature of real turbulence. The GOY model contains only one velocity mode per cascade level instead of infinitely many modes in real turbulence. This one-mode approximation leads to an overestimation of the fluctuation strength. In order to confirm this, we performed another simulation with more modes per level within the reduced REWA of the Navier-Stokes equation. This will be presented in Sec. III.

\section{Modulated driving force}

In this section we present further results within the GOY model based on a nonfluctuating driving force $F_{0}$ which is regularly modulated as was $\epsilon_{0}$ in the preceding section. This case may be more comparable to the experimental method in Ref. [5], because there the driving force is modulated. What cannot be modeled with GOY is the spatial inhomogeneity in the experiments [5].

In Eqs. (2) we now apply a forcing

$$
F=F(t)=F_{0}\left(1+e_{f} \sin \omega t\right),
$$

with a modulation amplitude of $e_{f}=0.2$. As in Sec. II B, we calculate the ensemble averaged time series of the energy input rate $e_{i n}(t)$, see first line of Eq. (4), and the total energy of the system $E(t)$, cf Eq. (5), for 89 different frequencies between $0.0144 \leqslant \omega \tau_{L} \leqslant 3.04$, again logarithmically equally distributed. The normalized energy $E^{\text {norm }}(t)$ and energy input rate $e_{i n}^{\text {norm }}(t)$ are then fitted by a function according to Eq. (7), with the parameters $E_{\text {const }}, A, \Phi$ and $e_{i n, \text { const }}, A_{e_{i n}}$, $\Phi_{e_{i n}}$, respectively.

The amplitudes $A$ and $A_{e_{i n}}$ are plotted in Fig. 3 as a function of the dimensionless frequency $\omega \tau_{L}$. Also in this case of a modulated force, the response amplitude is almost constant for small frequencies, namely, $A \simeq 1$, and decreases as $1 / \omega$ for high frequencies, see Fig. 3(a) (full dots). Again, the long-dashed line represents the low-frequency limit of the mean-field theory for $A$ (which is $A \simeq 1$ in this case) and the dotted line represents the high-frequency limit. As in the preceding section, the crossover frequency between the two regimes is determined by the energy transfer time, i.e., $\omega_{\text {cross }} \tau_{L} \simeq 1 / a \simeq 0.40$ (as $a=2.48$ in this case), which is marked by the small arrow in Fig. 3(a). The amplitude of the energy input rate $A_{e_{i n}}$ starts with a value of about 1.5 for low frequencies and merges towards 1 for high frequencies, see Fig. 3(c). This indicates that at very large frequencies the velocity is not oscillating anymore as it only feels a mean constant force. The oscillations of the energy input rate are then only a consequence of the oscillation of the driving force $F$. In the mean-field theory we have observed the same trend for both amplitudes. The corresponding mean-field results are included as dashed lines.

Both amplitudes $A$ and $A_{e_{i n}}$ show a maximum at a frequency near the crossover frequency $\omega \tau_{L} \simeq \tau_{L} / \tau=a^{-1}$ $=0.40$. In the compensated plot Fig. 3(b), where $A /\left(\omega \tau_{L}\right)^{-1}$ is plotted as a function of frequency, a clear deviation from the dotted line representing $A \propto 1 / \omega$ can be observed. At this frequency, the mean-field theory predicts a first maximum for the energy response amplitude [Fig. 3(a)] and a maximum directly followed by a minimum for the energy input rate, see Fig. 3(c). This frequency is again connected to the energy transfer time $\tau$. As in the case of Sec. II B, further extrema are washed out due to the strong fluctuations of the GOY dynamics.

\section{MODULATED TURBULENCE IN A REDUCED WAVE VECTOR SET APPROXIMATION}

As was pointed out in Sec. I, a full numerical simulation of the Navier-Stokes equation for modulated turbulence at 
high Reynolds numbers is still not possible or requires low Reynolds numbers. Therefore, we first have considered the GOY shell model. This model correctly describes many features of turbulence, however, due to the one-mode approximation in each cascade level, it contains various artifacts. Namely, it strongly overestimates the strength of the fluctuations. The aim of this section is to study the problem of modulated turbulence within another model, the REWA [1517], which is much closer to the Navier-Stokes equation than the GOY model and contains much more modes per cascade level. Of course, as compared to full numerical simulations of the Navier-Stokes equation, it still contains a mode reduction in order to make the computational effort reasonable for the desired high Reynolds numbers. The present approximation has been introduced and extensively studied in Refs. [15-17]. Here, we use it together with a time-dependent driving. For completeness, we very briefly explain the approximation before we present the results with modulated driving.

\section{A. The reduced wave vector set approximation}

The velocity field $\boldsymbol{u}(\boldsymbol{x}, t)$ is Fourier transformed into plane waves, $\boldsymbol{u}(\boldsymbol{x}, t)=\Sigma_{p} \boldsymbol{u}(\boldsymbol{p}, t) e^{i \boldsymbol{p} \cdot \boldsymbol{x}}$. Periodic boundary conditions are applied on a periodicity volume $\left(2 \pi L_{0}\right)^{3}$. The wave vectors $\boldsymbol{p}$ are given by $\boldsymbol{p}=\left(p_{i}\right)=\left(n_{i} L_{0}^{-1}\right)$, with $n_{i}=0, \pm 1$, $\pm 2, \ldots$. In order to efficiently deal with the large number of modes involved, the reduced wave vector set approximation selects a limited number of modes by admitting only a geometrically scaling subset $K=\cup_{l} K_{l}$ of wave vectors, i.e., $\boldsymbol{u}(\boldsymbol{x}, t)=\sum_{\boldsymbol{p} \in K} \boldsymbol{u}(\boldsymbol{p}, t) e^{i \boldsymbol{p} \cdot \boldsymbol{x}}$. On this subset $K=\left\{\boldsymbol{p}_{n}^{(l)}, n\right.$ $\left.=1, \ldots, N, l=0, \ldots, l_{\max }\right\}$ the Navier-Stokes equation for incompressible flow,

$$
\begin{aligned}
\frac{d}{d t} u_{i}\left(\boldsymbol{p}_{n}^{(l)}\right)= & -\nu\left(\boldsymbol{p}_{n}^{(l)}\right)^{2} u_{i}\left(\boldsymbol{p}_{n}^{(l)}\right)+f_{i}\left(\boldsymbol{p}_{n}^{(l)}\right) \\
& -i M_{i j k}\left(\boldsymbol{p}_{n}^{(l)}\right) \sum_{\boldsymbol{q}_{1}, \boldsymbol{q}_{2} \in K, \boldsymbol{q}_{1}+\boldsymbol{q}_{2}=\boldsymbol{p}_{n}^{(l)}} u_{j}\left(\boldsymbol{q}_{1}\right) u_{k}\left(\boldsymbol{q}_{2}\right),
\end{aligned}
$$

together with the continuity equation, $\boldsymbol{p}_{n}^{(l)} \cdot \boldsymbol{u}\left(\boldsymbol{p}_{n}^{(l)}\right)=0$, is solved. $M_{i j k}$ is the coupling matrix, $M_{i j k}(\boldsymbol{p})=\frac{1}{2}\left[p_{j} P_{i k}^{\perp}(\boldsymbol{p})\right.$ $\left.+p_{k} P_{i j}^{\perp}(\boldsymbol{p})\right]$, where $P_{i j}^{\perp}(\boldsymbol{p})$ is the orthogonal projector to $\boldsymbol{p}$. The subset $K$ consists of a basic subset $K_{0}=\left\{\boldsymbol{p}_{n}^{(0)}, n\right.$ $=1, \ldots, N\}$ together with its scaled replicas $\boldsymbol{p}_{n}^{(l)}=2^{l} \boldsymbol{p}_{n}^{(0)}, l$ $=1, \ldots, l_{\max }$. In the present simulation we take $N=74$ wave vectors $\boldsymbol{p}_{n}^{(0)}$. The wave vectors $\cup_{l} K_{l}$ are chosen such that they span a wide range of length scales, but still dynamically interact to a good degree. The largest eddies of the order $L_{0}$ are represented by the wave vectors in $K_{0}$, whereas the subsets $K_{l}$ contain wave vectors of smaller and smaller eddies. The choice of the smallest eddies, i.e., the value of $l_{\max }$, depends on the kinematic viscosity $\nu . l_{\max }$ and $\nu$ are adjusted such that the velocity amplitudes $\boldsymbol{u}\left(\boldsymbol{p}_{n}^{\left(l_{\max }\right)}, t\right)$ of the smallest eddies are almost zero. In this simulation, $\nu$ is chosen as $\nu=5 \times 10^{-5}$ and the number of levels as $l_{\max }+1$ $=9$.
To maintain the turbulent flow we apply a forcing as in Refs. [15-17]:

$$
\boldsymbol{f}(\boldsymbol{p}, t)= \begin{cases}\epsilon_{0} \frac{\boldsymbol{u}(\boldsymbol{p}, t)}{\sum_{\boldsymbol{q} \in K_{i n}}|\boldsymbol{u}(\boldsymbol{q}, t)|^{2}}(1+e \sin \omega t), & \boldsymbol{p} \in K_{\text {in }} \\ 0, & \boldsymbol{p} \notin K_{\text {in }} .\end{cases}
$$

The subset $K_{\text {in }}$ of $K_{0}$ by choice contains the 14 wave vectors with the three smallest lengths. In Ref. [16] it has been shown that the statistics of the solutions of the equations of motion do not depend on the particular choice of $K_{0}$. This forcing corresponds to the same type of forcing, which has already been applied to the GOY model in Sec. II B. It enforces the energy input rate to be modulated:

$$
e_{i n}=\left\langle\left\langle\sum_{p_{n}^{(l)} \in K} u^{*}\left(p_{n}^{(l)}\right) \cdot f\left(p_{n}^{(l)}\right)\right\rangle=\epsilon_{0}(1+e \sin \omega t) .\right.
$$

Equation (9) is a set of $3 N\left(l_{\max }+1\right)$ coupled ODEs for the complex mode amplitudes $u_{i}\left(\boldsymbol{p}_{n}^{(l)}\right)$ which is numerically solved. Length scales are measured in units of $L_{0}$ and time scales in units of $L_{0}^{2 / 3} \epsilon_{0}^{-1 / 3}$. A Reynolds number can be defined as follows. The wavelength $\lambda$ of the smallest wave vector gives an external length scale $L=2 \pi / \sqrt{6}$, and a typical velocity on that scale is determined by the rms of one velocity component, $u_{1, r m s}$. Then, in our case, the Reynolds number is $\operatorname{Re}=u_{1, r m s} L / \nu=1.234 \times 10^{5}$ because from the simulations we obtain $u_{1, r m s}=2.405$.

The main features of fully developed turbulence as irregular velocity signals, characteristic scaling of structure functions, etc., are well described within this approximation, as has been shown in Refs. $[15,16,22]$. The REWA solutions show small scale intermittency, which is produced by the competition between down-scale energy transport and viscous dissipation on the small scales [16,22]. Other mechanisms leading to intermittency in turbulence as, e.g., nonlocal interactions between wave vectors are underestimated in this approximation [23]. The down-scale energy transport in the REWA fluid is less effective than in real turbulence, because in this approximation the larger wave vectors are more and more thinned out [24]. This is in contrast to the case of the complete set of wave vectors (e.g., in full grid simulations) where the density of states increases $\propto p^{2}$, whereas in the reduced wave vector set $K$ the number of admitted wave vectors decreases as $1 / p$ [17]. In Ref. [24] it has been shown that this reduced energy transport leads to an overestimation of the Taylor Reynolds number of the system as well as the Kolmogorov constant $b$, defined by $D(r)=b(\epsilon r)^{2 / 3}$, by roughly one order of magnitude for our choice of $N$. In the present simulation we obtain $b=83.5$ instead of $b=6-9$ as in experiments [25-27]. Since $D(L)$ is the energy density $\propto\left\langle\left\langle\boldsymbol{u}^{2}\right\rangle\right\rangle$ of the fluctuations in the fluid system, the large $b$ value indicates that in the REWA approximation the strength of the fluctuations is highly overestimated. The large Kol- 


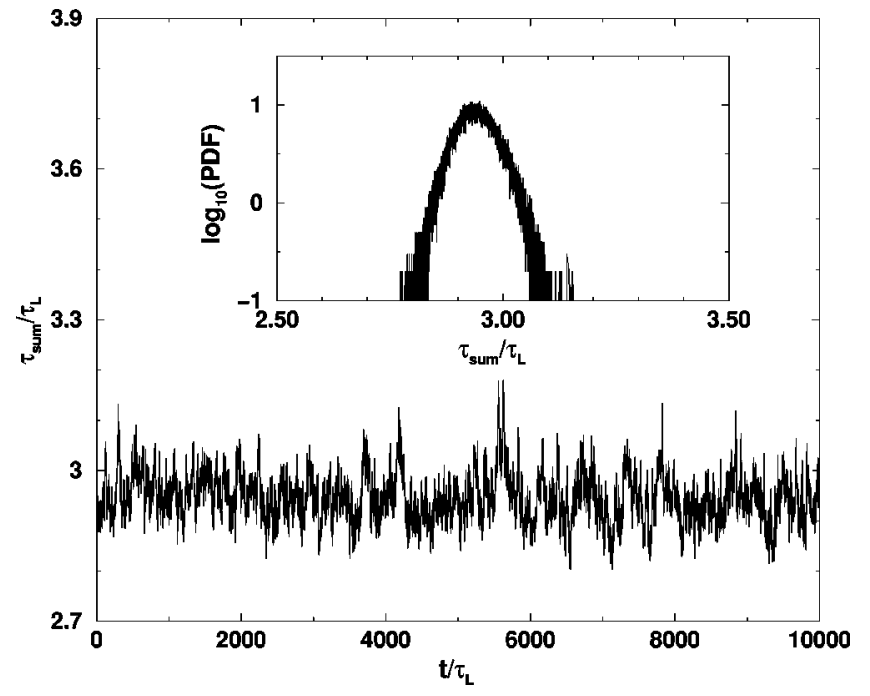

FIG. 7. Time series of the cascade time $\tau_{\text {sum }}(t)$ in REWA. Inset: probability distribution of $\tau_{\text {sum }} / \tau_{L}$. The fluctuations are considerably smaller than in the GOY model. Note the different scales in this figure and Fig. 6. The mean is $\tau_{\text {sum }} / \tau_{L}=a=2.94$, and the width $(F W H M) / 2=0.02 a$ giving about $2 \%$ fluctuations.

mogorov constant will change the relevant time scales in the system, as will be shown in Sec. III B.

The characteristic time scale for the turbulent energy transfer on scale $l$ can be estimated as $\tau(l)=1 /\left(p^{(l)} u_{r m s}^{(l)}\right)$, where $p^{(l)}$ denotes the mean wave number on scale $l$, i.e., it is the mean inverse eddy size in $K_{l}$. As in the GOY model, the time scale of viscous dissipation is $\tau_{d}=\left[\nu\left(p^{(l)}\right)^{2}\right]^{-1}$. Again, in the ISR $\tau(l)>\tau_{d}$, whereas in the VSR $\tau_{d}>\tau(l)$. From a simulation with stationary forcing, i.e., $e=0$ in Eq. (10), the time delay of the energy down-transport $\tau_{\text {sum }}$ is then estimated by the sum of all $\tau(l)$ in the ISR, $\tau_{\text {sum }}$ $=\Sigma_{l \in I S R} \tau(l) \simeq 0.186$. The largest of these $\tau(l)$, on the largest scale, $\tau(0)=0.0632$ can be regarded as a large eddy turnover time $\tau_{L}$. Thus, $\tau_{\text {sum }}=2.94 \tau_{L}$, and the factor between the cascade time scale and the large eddy turnover time is $\tau_{\text {sum }} / \tau_{L}=a=2.94$.

As we have seen in the GOY model, Fig. 6, the energy transfer time is strongly fluctuating. We attributed these strong fluctuations to the one-mode per level approximation of the GOY model. Figure 7 shows a time series of the energy transfer time $\tau_{\text {sum }}(t)$ [use $\tau(l)$ with $u^{(l)}(t)$ ] in the present REWA, and, in the inset, the distribution of this time scale. Clearly, the fluctuations are much weaker than in the GOY model; they are zero in the mean-field approximation. The distribution is centered around $\tau_{\text {sum }} / \tau_{L}=a$ with a width [(FWHM)/2] of about $0.02 a$.

\section{B. Modulated energy input rate}

The response of the system to a modulated driving force, cf. Eq. (10), is calculated now in terms of the total energy of the system

$$
E(t)=\frac{1}{2}\left\langle\left\langle\sum_{l=0}^{l=l_{\max }} \sum_{\boldsymbol{p} \in K_{l}}|\boldsymbol{u}(\boldsymbol{p}, t)|^{2}\right\rangle .\right.
$$

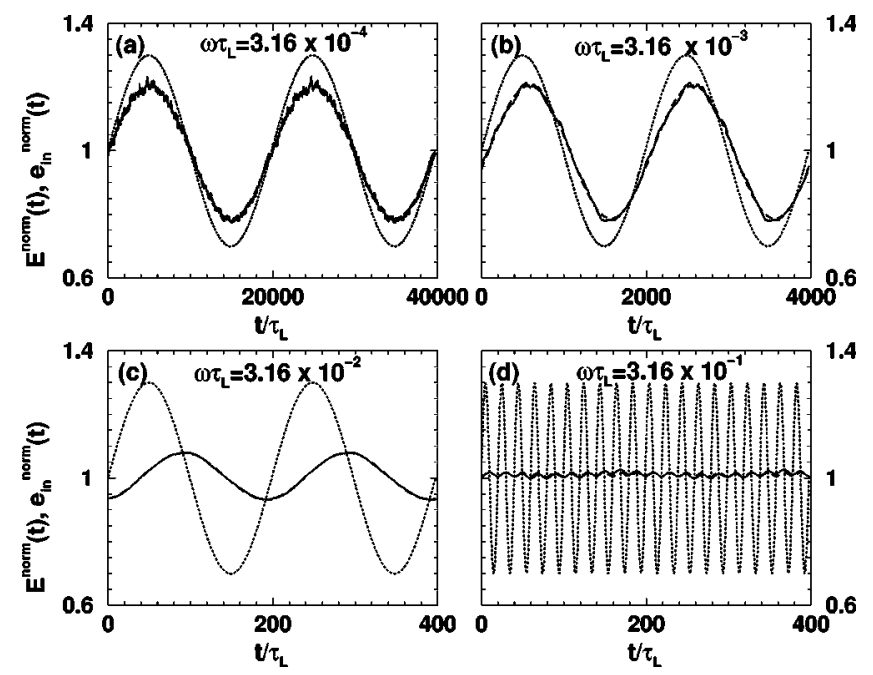

FIG. 8. Energy input rate $e_{i n}^{n o r m}=1+e \sin \omega t$ (dotted lines) and energy $E^{\text {norm }}$ (solid lines) for four different modulation frequencies $\omega$ as calculated in the REWA simulation. The energy input rate is modulated with a modulation amplitude of $30 \%$ of the constant energy input rate, i.e., $e=0.3$ in Eq. (10). Also included is the fit to the energy data cf. Eq. (7) as dashed lines but these are in all cases indistinguishable from the solid lines. The averaged time series of $E^{\text {norm }}$ are repeated once for better visibility. (a) $\omega \tau_{L}$ $=3.16 \times 10^{-4}$, (b) $\omega \tau_{L}=3.16 \times 10^{-3}$, (c) $\omega \tau_{L}=3.16 \times 10^{-2}$, (d) $\omega \tau_{L}=0.316$. For larger $\omega \tau_{L}$ the energy is indistinguishable from 1 on this scale. The crossover to the $1 / \omega$-decay regime is in this simulation at $\omega \tau_{L} \simeq 1.1 \times 10^{-2}$, between the frequencies of (b) and (c).

The modulation amplitude of the energy input rate $e_{i n}[\mathrm{Eq}$. (11)] is chosen as $e=0.3$. The response is calculated for 150 (approximately equally spaced on a logarithmic scale) frequencies between $0.00016 \leqslant \omega \tau_{L} \leqslant 3.0$. The energy is normalized by $E_{0}$, calculated from a stationarily forced solution with $e=0$ and averaged as $E$. The oscillating response $\Delta(t)$ of the system is defined in the same way as for the GOY model, see Eq. (6). Then, as for the GOY calculations, the averaged and normalized signals $E^{\text {norm }}(t)$ are fitted with Eq. (7). The fit parameter $E_{\text {const }}$ is again near 1 for all frequencies, $E_{\text {const }}=1.0064 \pm 0.0065$. In Fig. 8 the time averaged responses and the normalized energy input rates are plotted for four different driving frequencies. Also the fits according to Eq. (7) have been included as dashed lines but are indistinguishable from the solid lines for the energy signal itself.

We observe in Fig. 8 for REWA the same features as in Fig. 5 for the GOY model and in Fig. 1 of Ref. [3] for the mean-field model. For the two lower frequencies the response amplitude remains almost constant and is about $2 / 3$ of the amplitude of the energy input rate, whereas for the two higher frequencies the response amplitude strongly decreases. This trend becomes more clear in Fig. 2(a), where the amplitude of the response-determined from fit (7) - is shown as a function of the driving frequency (full dots). For low driving frequencies $A \simeq 2 / 3$, whereas for high frequencies the amplitude decreases as $1 / \omega$. The crossover between the regime of constant amplitude and the one of $1 / \omega$ decay takes place at $\omega_{\text {cross }} \tau_{L} \simeq 0.011$, i.e., at a much smaller fre- 
quency than expected from the original case of the meanfield theory in which the crossover was at $\omega_{\text {cross }}^{M F} \tau_{L}=1$ with $b=6$. We understand this as follows. In Sec. III A it was mentioned that the Kolmogorov constant in the REWA simulation is $b=83.5$ instead of $b=6-9$ as in experiments. In the figures of the mean-field approach [3] we have set $b=6$. The mean-field solution for a general $b$ revealed that the crossover frequency decreases with increasing $b$ while the positions of the response maxima are left unchanged. For $b$ $=83.5$ the mean-field crossover frequency is at $\omega_{\text {cross }}^{M F} \tau_{L}$ $=(6 / b)^{3 / 2} \simeq 0.019$ in close agreement to what we observe in the REWA simulations. The response amplitude calculated from the mean-field model with $b=83.5$ is included in Fig. 2(a) as dashed line. Apart from the changed crossover frequency we observe that, in the mean-field calculations, the first response maximum at $\omega \tau_{L} \simeq 0.1$ is considerably smaller and broadened as compared to the case with $b=6$, given as the dashed line in Fig. 1(a). In agreement with this, our REWA simulations (with a value $b=83.5$ ) show a broad maximum in the response amplitude at $\omega \tau_{L} \simeq 0.028$. This means it occurs at a similar frequency as the mean-field model. The maximum becomes more clear in the compensated plot, Fig. 2(b), where $A /\left(\omega \tau_{L}\right)^{-1}$ is shown as a function of frequency. There, we observe a deviation from the $1 / \omega$ decay of the amplitude by a factor 1.4 at the maximum in the REWA simulations. The mean-field maximum has a height of 2.8. The subsequent maxima and minima in the mean-field model occur at frequencies where the amplitude is already very small $\left(A \leqslant 10^{-2}\right)$ because the crossover to the $1 / \omega$ regime takes place at a much smaller frequency whereas the response maxima stay at the same frequencies as for a smaller $b$. Therefore, the higher order maxima are not visible in the REWA simulations. The cascade time shows about $2 \%$ fluctuations in REWA as shown in Fig. 7. However, at small response amplitudes these fluctuations are already large enough to wash out the higher-order response maxima.

In conclusion, the REWA system reproduces qualitatively the features of modulated turbulence as predicted by the mean-field model including the first response maximum. The latter is considerably weakened due to the large Kolmogorov constant in REWA. Another consequence of the large $b$ is that the crossover between constant amplitude and $1 / \omega$ decay is shifted towards smaller frequencies, and therefore the higher-order maxima and minima are already at very small amplitudes where the fluctuations in the cascade time scale are finally large enough to wash them out. We cannot clarify, at present, how close the response in direct numerical simulations-which lead to an order of magnitude smaller $b$ and thus have much smaller fluctuations-will come to the mean-field features, but we expect a clearly visible first maximum at least.

\section{CONCLUSIONS}

We have simulated the response of modulated turbulence within two numerical models. Namely, we have used the GOY shell model and the reduced wave vector approximation (REWA) of the Navier-Stokes equation. The results are compared with predictions from a mean-field theory. For a modulated energy input rate this mean-field theory had predicted a constant response amplitude for low frequencies and a $1 / \omega$ decay for high driving frequencies. In addition, at certain frequencies connected with the energy cascade time scale, a sequence of maxima and minima of the response amplitude is observed.

Both numerical models well reproduce the basic trend, i.e., the constant amplitude for small $\omega$ and the $1 / \omega$ decrease for large $\omega$. The main response maximum can be observed in both numerical models, although it is weakened due to fluctuations. The higher-order maxima and minima as predicted by the mean-field theory cannot be identified in the simulations. They are washed out by fluctuations. We believe that in real turbulence with a realistic Kolmogorov constant and only narrow fluctuations of the energy transfer time the first maximum should be clearly observable and possibly also the higher-order extrema in the response. Thus, the predictions of the mean-field model, which excludes all fluctuations, might be quite reasonable for real turbulence. To further study the response maxima numerically, it is necessary to perform full numerical simulations of the Navier-Stokes equation, as then all relevant time scales including their fluctuations are reproduced realistically, which turned out to be essential for the observation of the response maxima.

Recent experiments on modulated turbulence [5] revealed evidence for the response maxima. These experiments may be more comparable to the case of a modulated force instead of a modulated energy input rate. We have studied this case also within the mean-field model and have found basically the same behavior of the energy response as for a modulated energy input rate. In addition, the amplitude of the energy input rate showed "wiggles" at the same frequencies where the energy response had maxima. In the experiments the response maxima were measured in the energy input rate, which can be regarded as a response of the system as well in this case. Also the constant amplitude for low driving frequencies and the $1 / \omega$ decay of the velocity response-which in leading order is corresponding to a $1 / \omega$ decay of the energy response, as well-have been observed in the experiments. Here, we have studied the case of a modulated driving force within the GOY shell model. Also in this simulation, the response amplitude behaves basically as in the mean-field model, i.e., it decreases as $1 / \omega$. The energy response amplitude as well as the amplitude of the energy input rate show the main maximum.

There are two regimes in the frequency behavior of the response amplitude, namely, a constant amplitude at low $\omega$ and a decreasing amplitude at high $\omega$. The present simulations give further confidence that the crossover frequency between these two regimes gives the correct order of magnitude of the cascade time scale, i.e., in experiments it can be used to measure this time scale as suggested in Ref. [5].

Both models in the present study were able to reproduce the main features of the frequency behavior of the response amplitude in modulated turbulence as predicted by the meanfield model, however, both also have their shortcomings, which prevent us from correctly predicting the behavior of real turbulence in all quantitative details. Therefore, we believe that it is worthwhile to further study modulated turbulence numerically as well as experimentally. 


\section{ACKNOWLEDGMENTS}

The work is part of the research program of the Stichting voor Fundamenteel Onderzoek der Materie (FOM), which is financially supported by the Nederlandse Organisatie voor
Wetenschappelijk Onderzoek (NWO). This research was also supported by the German-Israeli Foundation (GIF) and by the European Union under Contract No. HPRN-CT-200000162.
[1] S.B. Pope, Turbulent Flows (Cambridge University Press, Cambridge, 2000).

[2] U. Frisch, Turbulence (Cambridge University Press, Cambridge, 1995).

[3] A. von der Heydt, S. Grossmann, and D. Lohse, Phys. Rev. E 67, 046308 (2003).

[4] H. Effinger and S. Grossmann, Z. Phys. B: Condens. Matter 66, 289 (1987).

[5] O. Cadot, J.H. Titon, and D. Bonn, J. Fluid Mech. 485, 161 (2003).

[6] E.B. Gledzer, Sov. Phys. Dokl. 18, 216 (1973).

[7] M. Yamada and K. Ohkitani, J. Phys. Soc. Jpn. 56, 4210 (1987).

[8] M. Yamada and K. Ohkitani, Prog. Theor. Phys. 79, 1265 (1988).

[9] K. Ohkitani and M. Yamada, Prog. Theor. Phys. 81, 329 (1989).

[10] L. Kadanoff, D. Lohse, J. Wang, and R. Benzi, Phys. Fluids 7, 617 (1995).

[11] L. Biferale, Annu. Rev. Fluid Mech. 35, 441 (2003).

[12] M.H. Jensen, G. Paladin, and A. Vulpiani, Phys. Rev. A 43, 798 (1991).

[13] T. Bohr, M.H. Jensen, G. Paladin, and A. Vulpiani, Dynamical Systems Approach to Turbulence (Cambridge University Press,
Cambridge, 1998).

[14] J.O. Hooghoudt, D. Lohse, and F. Toschi, Phys. Fluids 13, 2013 (2001).

[15] J. Eggers and S. Grossmann, Phys. Fluids A 3, 1958 (1991).

[16] S. Grossmann and D. Lohse, Z. Phys. B: Condens. Matter 89, 11 (1992).

[17] S. Grossmann and D. Lohse, Phys. Fluids 6, 611 (1994).

[18] W. Press, S. Teukolsky, W. Vetterling, and B. Flannery, Numerical Recipes (Cambridge University Press, Cambridge, 1986).

[19] R. Benzi, L. Biferale, and G. Parisi, Physica D 65, 163 (1993).

[20] N. Schörghofer, L. Kadanoff, and D. Lohse, Physica D 88, 40 (1995).

[21] L. Kadanoff, D. Lohse, and N. Schörghofer, Physica D 100, 165 (1997).

[22] S. Grossmann and D. Lohse, Physica A 194, 519 (1993).

[23] S. Grossmann, D. Lohse, and A. Reeh, Phys. Rev. Lett. 77, 5369 (1996).

[24] S. Grossmann and D. Lohse, Phys. Rev. E 50, 2784 (1994).

[25] A.S. Monin and A.M. Yaglom, Statistical Fluid Mechanics (The MIT Press, Cambridge, MA, 1975).

[26] K.R. Sreenivasan, Phys. Fluids 7, 2778 (1995).

[27] K.R. Sreenivasan and R.A. Antonia, Annu. Rev. Fluid Mech. 29, 435 (1997). 